\title{
Visual Servoing of an Under-Actuated Dynamic Rigid-Body System: An Image-Based Approach
}

\author{
Tarek Hamel and Robert Mahony
}

\begin{abstract}
A new image-based control strategy for visual servoing of a class of under-actuated rigid body systems is presented. The proposed control design applies to "eye-in-hand" systems where the camera is fixed to a rigid body with actuated dynamics. The control design is motivated by a theoretical analysis of the dynamic equations of motion of a rigid body and exploits passivity-like properties of these dynamics to derive a Lyapunov control algorithm using robust backstepping techniques. The proposed control is novel in considering the full dynamic system incorporating all degrees of freedom (albeit for a restricted class of dynamics) and in not requiring measurement of the relative depths of the observed image points. A motivating application is the stabilization of a scale model autonomous helicopter over a marked landing pad.
\end{abstract}

Index Terms-Image-based visual servo (IBVS), nonlinear control, rigid-body dynamics, under-actuated systems.

\section{INTRODUCTION}

$\mathbf{V}$ ISUAL servoing algorithms have been extensively developed in the robotics field over the last ten years. Visual servo systems may be divided into two main classes [17]: position-based visual servo (PBVS) involves reconstruction of the target pose with respect to the robot and leads to a Cartesian motion planning problem [33]. Image-based visual servo (IBVS) aims to control the dynamics of features in the image plane directly [31], [7]. The Cartesian motion planning task is implicitly resolved via minimization of an image-based error function [25], [22]. IBVS methods offer advantages in robustness to camera and target calibration errors, reduced computational complexity, and simple extension to applications involving multiple cameras compared to PBVS methods [11]. However, classical IBVS suffers from two key problems. Firstly, it is necessary to determine the depth of each visual feature used in the image error criterion independently from the control algorithm. Secondly, the rigid-body dynamics of the camera ego-motion are highly coupled when expressed as target motion in the image plane. Various approaches to overcoming the relative depth requirement for classical IBVS have been reported, including; estimation via partial pose estimation [17], adaptive control [20], and estimation of the image Jacobian using quasi-Newton techniques [10], [21]. Recent work has tended to concentrate on hybrid control methods whereby translational and attitude control

Manuscript received June 6, 2000; revised June 20, 2001. This paper was recommended for publication by Associate Editor P. Allen and Editor S Hutchinson upon evaluation of the reviewers' comments.

T. Hamel is with CEMIF-SC, Université d'Evry, CE 1455 Courcouronnes, France (e-mail: thamel@iup.univ-evry.fr).

R. Mahony is with the Department of Engineering, Australian National University, Canberra, ACT 0200 Australia (e-mail: mahony@ieee.org).

Publisher Item Identifier S 1042-296X(02)04297-0. are treated separately [17], [19], [6], [5]. This approach also addresses the problems associated with the coupled nature of the camera ego-motion in the image plane. Classical visual servo control was principally developed for serial-link robotic manipulators [11]. In such situations, the dynamics of the system are easily compensated using a computed torque (or high gain) control design and the visual servo control may be derived from a first-order model of the image dynamics [7]. Recent applications in high-performance systems and under-actuated dynamic systems have lead researchers to consider full dynamic control design. Coupling of the camera ego-motion dynamics in the image plane proves to be a significant obstacle in achieving this goal. Kelly [12] proposed an asymptotically stable method for position regulation for fixed-camera visual servoing for a dynamic system. Work by Zergeroglu et al. [35] has used robust backstepping techniques to deal with the full dynamics of a planar robot for a visual servo control, while Maruyama et al. [18] have investigated dissipative control strategies. A further complication is encountered when an under-actuated dynamic system is used as the platform for the camera. Zhang and Ostrowski [36] used a Lagrangian representation of the system dynamics to obtain an IBVS control for a blimp, an under-actuated nonholonomic system. Applications in flight control of UAVs are a strong driving force in this area and several authors have investigated PBVS methods for such systems [1], [27]. In addition to the classical visual servo approach, some work has been done to apply biomimetic control design to UAVs. Examples are visual flow-based control algorithms [30], [3] and feature detection [32].

In this paper, we propose an IBVS algorithm for a class of under-actuated dynamic systems. The proposed algorithm does not require accurate depth information for observed image features. The closed-loop system displays the robustness to camera and target calibration errors and reduced computational complexity characteristic of IBVS methods. The design is based on a theoretical analysis of the dynamic equations of motion of a rigid body and exploits structural passivity-like properties of these dynamics to derive a Lyapunov control algorithm using robust backstepping techniques. This approach overcomes some of the difficulties associated with the highly coupled dynamics of the camera ego-motion in the image dynamics. Simulation of the closed-loop response of the example studied indicates strong robustness properties derived from the Lyapunov stability criterion and the centroid visual features used. In order to implement the proposed servo algorithm, separate measurements of linear and angular velocity are needed. These measurements are not used in classical visual servo design since the kinematic velocities are implemented directly as the control variables. In ad- 
dition, the proposed algorithm requires a single inertial direction to be measured in order to define the image error criterion. This information must be obtained using a routine separate to the control algorithm, analogous in a sense to the role of image depth measurement for classical IBVS control design. In writing this paper, the authors are strongly motivated by the application of visual servo control to the regulation of hover maneuvers for VTOL robotic aircraft. A vision system is a natural choice for a cheap, passive and adaptable sensor that can be used to regulate the motion of such UAVs. For these systems, there are a host of cheap, light INS systems that are being developed that will provide sufficiently accurate measurements of angular and linear velocity for implementation of the proposed control. The inertial direction used for most UAV applications requiring hover maneuvers is the vertical axis. This is often directly supplied by an INS system or can be obtained by filtering the accelerometer output.

The paper is arranged into five sections. Section II presents the fundamental equations of motion for an autonomous robot with a single rigid component, demonstrates their passivity-like properties and shows that these properties are present in the image space. Section III introduces the image space error representation and how it can be rewritten in a form that is more amenable to control design and analysis. Section IV derives a Lyapunov control for under-actuated systems based on a robust backstepping design. Section V applies the visual control strategy to an idealized dynamic model of a reduced scale helicopter and presents some simulation results.

\section{Visual Dynamics of a Point TARget AND PASSIVITY-LIKE PROPERTIES}

In this section, a general dynamic model of a rigid body evolving in $\mathbb{R}^{3}$ is presented. These dynamics define the ego-motion of the camera and are used to derive the observed dynamics of a stationary target.

Denote the rigid body by the letter $\mathbf{A}$. Let $\mathcal{I}=\left\{E_{x}, E_{y}, E_{z}\right\}$ denote a right-hand inertial frame such that $E_{z}$ denotes the vertical direction downwards into the earth. The position of the rigid body is measured at the focal point of the camera rather than the center of mass of the body and is denoted $\xi=(x, y, z) \in \mathcal{I}$. Let $\mathcal{A}=\left\{E_{1}^{a}, E_{2}^{a}, E_{3}^{a}\right\}$ be a (right-hand) body fixed frame for $\mathbf{A}$ (cf. Fig. 1). The attitude of $\mathbf{A}$ is given by the rotation $R: \mathcal{A} \rightarrow \mathcal{I}$, where $R \in S O(3)$ is an orthogonal rotation matrix.

Let $V$ and $\Omega \in \mathcal{A}$ denote the body fixed frame linear and angular velocities of the rigid body. Let $m$ denote the mass of $\mathbf{A}$ and let $\mathbf{I} \in \mathbb{R}^{3 \times 3}$ denote the constant inertia matrix around the focal point of the camera (expressed in the body fixed frame $\mathcal{A}$ ). Newton's equations of motion (cf. for example [8]) yield the following dynamic model for the motion of a rigid object:

$$
\begin{aligned}
\dot{\xi} & =R V \\
m \dot{V} & =-m \Omega \times V+F \\
\dot{R} & =R \operatorname{sk}(\Omega), \\
\mathbf{I} \dot{\Omega} & =-\Omega \times \mathbf{I} \Omega+\Gamma
\end{aligned}
$$

where $F \in \mathcal{A}$ combines all the external linear forces acting on $\mathbf{A}$ (including gravity) and $\Gamma$ combines all external torques.

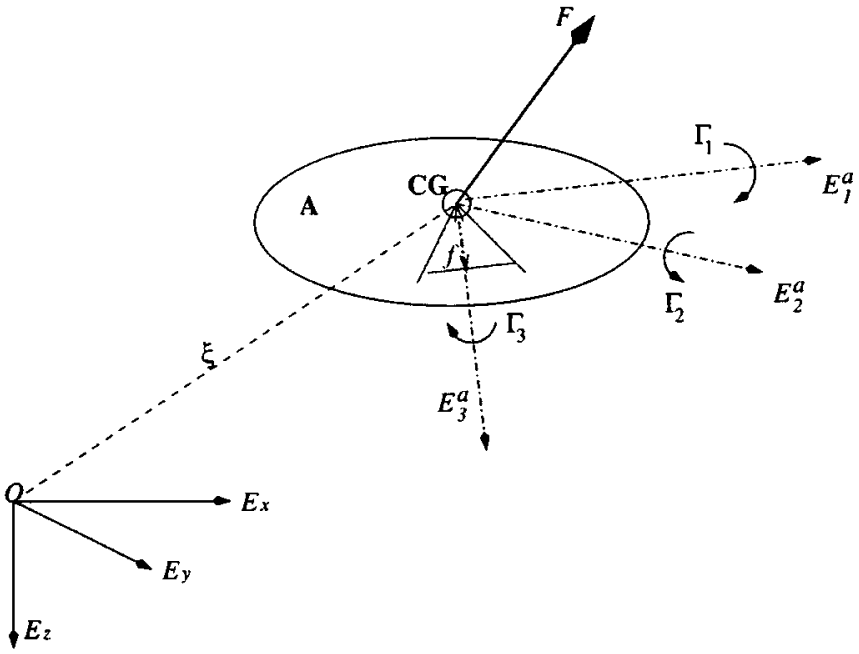

Fig. 1. Rigid body with force and torque control.

The notation $\operatorname{sk}(\Omega)$ denotes the skew-symmetric matrix such that $\operatorname{sk}(\Omega) v=\Omega \times v$ for the vector cross-product $\times$ and any vector $v \in \mathbb{R}^{3}$.

The class of under-actuated dynamic systems considered are those for which the force input $F$ incorporates a single independent actuator while full actuation of the torque $\Gamma$ is available. The force $F \in \mathcal{A}$ may be written

$$
F:=-u \mathbf{F}+m g R^{T} e_{3}
$$

where $\mathbf{F} \in \mathcal{A}$ is a constant unit vector in the body fixed frame representing the fixed orientation of the thruster or actuator and $m g R^{T} e_{3}$ is the gravitational force. The control input $u \in \mathbb{R}$ represents the magnitude of thrust applied. Section V presents the structure for an idealized helicopter.

Equations (1)-(4) have an important cascade structure. Expressing (1) and (2) in the inertial frame where $v=R V$ and $f=R F$ the translation dynamics are a simple linear cascade

$$
\dot{\xi}=v, \quad m \dot{v}=f .
$$

These linear dynamics, along with the attitude dynamics equations (3) and (4), form a cascade system in triangular form [14]. Such systems may be feedback linearized and are in a suitable form for the application of backstepping and other nonlinear design techniques. This structure is the basis of many of the nonlinear tracking control algorithms developed for the helicopter [29], [13], [28], [16]. The cascade structure of the system leads to internal passivity-like properties (from virtual input to the backstepping error) typical of each iteration of backstepping control designs [14]. A key contribution of this paper is to show that these passivity-like properties can be recovered for a centroid image feature as long as a spherical camera geometry is used.

Let $\bar{P}_{i} \in \mathcal{I}$ for $i=1, \ldots, n$ represent $n$ stationary point targets. Let $P_{i} \in \mathcal{A}$ denote the coordinates of each $\bar{P}_{i}$ in the body fixed frame

$$
P_{i}=R^{T}\left(\bar{P}_{i}-\xi\right) .
$$

The geometry of the camera is modeled by its image surface $\mathcal{S}$ relative to its focal point. The image feature observed by the 


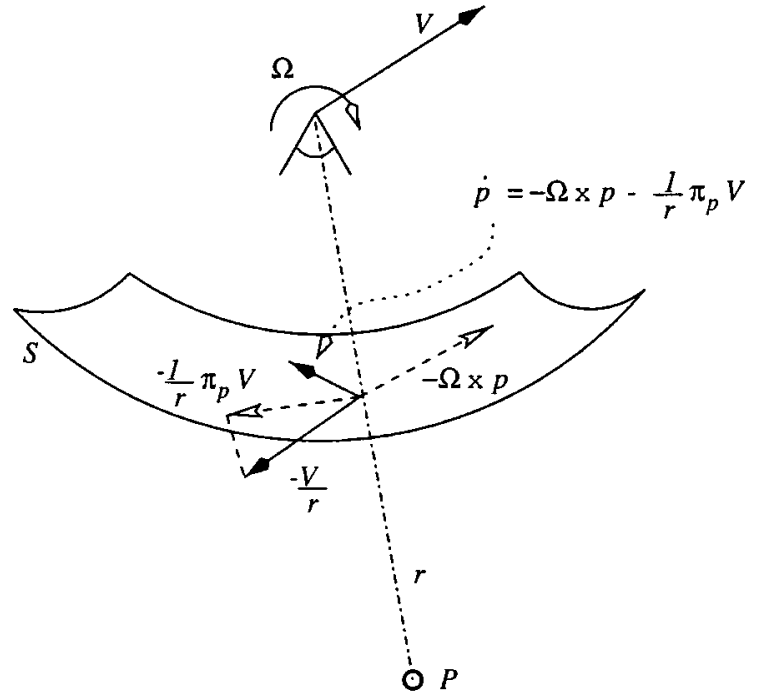

Fig. 2. Image dynamics for spherical camera geometry.

camera is denoted $p_{i}$ and is the projection of $P_{i}$ onto $\mathcal{S}$ in the body fixed frame (cf. Fig. 2)

$$
p_{i}=\frac{1}{r\left(P_{i}\right)} P_{i}
$$

where $r\left(P_{i}\right)$ is the relative depth of the target.

For a typical digital camera with a flat image plane then $p_{i}$ is given by the perspective projection

$$
p_{i}=\frac{f}{Z_{i}} P_{i}=\left(\begin{array}{c}
f \frac{X_{i}}{Z_{i}} \\
f \frac{Y_{i}}{Z_{i}} \\
f
\end{array}\right)
$$

where $P_{i}=\left(X_{i}, Y_{i}, Z_{i}\right) \in \mathcal{A}$, and $f>0$ is the focal length of the camera. Since the third entry of $p_{i}$ is constant it is common to use only the first two coordinates (corresponding to pixel position) to represent image points. In the following development the spherical projection is used extensively. If the image surface is spherical, then $r\left(P_{i}\right)=\left|P_{i}\right| / f$ which rescales the point $P_{i}$ onto the sphere of radius $f$. An arbitrary rescaling function $r\left(P_{i}\right),(6)$ enables one to consider general camera geometry.

Let

$$
\eta_{P_{i}}=\frac{\partial r}{\partial P_{i}}\left(P_{i}\right)
$$

be the differential of the scaling function $r$. Applying the chain rule, one has

$$
\frac{d}{d t} r\left(P_{i}\right)=\eta_{P_{i}}^{T} \dot{P}_{i}
$$

Note that $\mathcal{S}$ is a level surface of $r$ and that $\eta_{p_{i}}$ is normal to the surface for $p_{i} \in \mathcal{S}$.

Define $V_{i} \in \mathcal{A}$ to be the observed velocity of the target point $P_{i}$ represented in the body fixed frame

$$
V_{i}:=R^{T}\left(\dot{\bar{P}}_{i}-\dot{\xi}\right)
$$

Note that $V_{i}=-V$ (the body fixed frame velocity of the object) if and only if the inertial velocity of the target point $\dot{\bar{P}}_{i}=0$ is zero. The dynamics of the image point are

$$
\begin{aligned}
\dot{p}_{i} & =\frac{1}{r\left(P_{i}\right)} \dot{P}_{i}-\frac{\frac{d}{d t} r\left(P_{i}\right)}{r\left(P_{i}\right)^{2}} P_{i} \\
& =-\operatorname{sk}(\Omega) p_{i}+p_{i} \eta_{p_{i}}^{T} \operatorname{sk}(\Omega) p_{i}+\left(I_{3}-p_{i} \eta_{p_{i}}^{T}\right) \frac{V_{i}}{r\left(P_{i}\right)} .
\end{aligned}
$$

Due to the rotational ego-motion of the camera, (8) involves the angular velocity $\Omega$ as well as the velocity $V$. This dependence destroys the explicit triangular cascade structure of rigid body motion expressed in the inertial frame. However, under certain conditions on the camera geometry, it is possible to recover a passivity-like property (from virtual input to the backstepping error) sufficient to apply a backstepping control design [14]. The storage function considered is $p_{i}^{T} p_{i}$ and a (bilinear) supply rate $p_{i}^{T} Q V_{i}$ is used where $Q \geq 0$ is positive semi-definite. Section IV shows how this structure can be exploited in a backstepping control design.

In order that the image dynamics display the desired passivity-like properties it is necessary that the first two terms on the right-hand-side of (8) do not contribute to the derivative of the storage function. Thus, one requires that

$$
-p_{i}^{T} \operatorname{sk}(\Omega) p_{i}+p_{i}^{T} p_{i} \eta_{p_{i}}^{T} \operatorname{sk}(\Omega) p_{i}=\left(p_{i}^{T} p_{i}\right) \eta_{p_{i}}^{T} \operatorname{sk}(\Omega) p_{i}=0
$$

for all $\Omega$. This will hold only if $\eta_{p_{i}}^{T} \operatorname{sk}(\Omega) p_{i}=0$ for all $\Omega$ and imposes the constraint

$$
\eta_{p_{i}}:=\lambda\left(P_{i}\right) p_{i}
$$

where $\lambda(P)$ is some scalar function. Moreover, the vector $\eta_{P}$ is obtained as the gradient of a functional $r: \mathbb{R}^{3} \rightarrow \mathbb{R}$ and integrability results ensure that the only scaling function $r$ which satisfies (9) is $r(P)=|P| / f$, where $f>0$ is a constant. That is, the only image geometry that preserves the passivity-like properties of the body fixed frame dynamics of a rigid object in the image space are those of a spherical camera. Without loss of generality we choose $f=1$. In this case, $\eta_{p}=p$. The dynamics of an image point for a spherical camera of image surface radius unity are

$$
\dot{p}_{i}=-\operatorname{sk}(\Omega) p_{i}+\frac{\pi_{p_{i}}}{r\left(P_{i}\right)} V_{i}
$$

where $\pi_{p}=\left(I_{3}-p p^{T}\right)$ is the projection $\pi_{p}: \mathbb{R}^{3} \rightarrow T_{p} S^{2}$ onto the tangent space of the sphere $S^{2}$ at the point $p \in S^{2}$.

Remark II.1: It is not necessary to physically implement a spherical camera. It is sufficient to numerically compute the spherical projection of the observed image coordinates. For example, in the case of a pinhole camera, the data available for a point image are $p_{i}^{p}=\left(u_{i}, v_{i}, f\right)=\left(f X_{i} / Z_{i}, f Y_{i} / Z_{i}, f\right)$ expressed in the perspective projection of the coordinates of a point $P_{i}$ [cf. (7)]. The spherical coordinates for this image point are given by the algebraic transformation

$$
p_{i}=\frac{p_{i}^{p}}{\left|p_{i}^{p}\right|} .
$$

Motivated by the preceding discussion, we make the following assumptions. 


\section{Assumption II.2:}

1) The image surface of the camera is spherical with unit image radius $(f=1)$.

2) The target points are stationary in the inertial frame.

Given assumptions II.21) and II.22), the dynamics of an ensemble of $n$ observed image points $\left\{p_{1}, \ldots, p_{n}\right\}$ are given by

$$
\dot{p}_{i}=-\Omega \times p_{i}-\frac{\pi_{p_{i}}}{r_{i}} V, \quad \text { for } i=1, \ldots, n
$$

where $V=-V_{i}$ is the velocity of the rigid object and $r_{i}:=$ $r\left(P_{i}\right)$.

\section{IMAGE SPACE ERROR CRITERIA}

In this section, the image space error considered is defined.

Intuitively, the objective of a visual servo algorithm in image space is to match the observed image to a known "model" image of the target. Define a set of desired visual features $\left\{p_{1}^{*}, \ldots, p_{n}^{*}\right\}$ which correspond to the expected image of the target if the camera were placed in the desired position and attitude. Thus, $\left\{p_{1}^{*}, \ldots, p_{n}^{*}\right\}$ are a set of $n$ points on the spherical image plane. The full image space error term is a $3 n$-dimensional vector

$$
\delta=\operatorname{vect}\left(p_{i}-p_{i}^{*}\right) \in \mathbb{R}^{3 n} .
$$

In classical visual servo algorithms, the desired visual features are chosen to be fixed relative to the body fixed frame. As long as there are at least four point targets visible, minimizing $\delta$ ensures a particular attitude of the camera relative to the target [24], [9]. In this paper, we choose the orientation of the desired visual features $p_{i}^{*}$ to be fixed relative to the inertial frame. Since the orientation of the desired features is fixed in the inertial frame they inherit dynamics in the body fixed frame due to the rotational ego-motion of the camera

$$
\dot{p}_{i}^{*}=-\Omega \times p_{i}^{*} .
$$

The advantage of choosing the orientation of the desired visual targets to be fixed in the inertial frame is seen when the first order dynamics of the error are computed. Let

$$
\Pi:=\left(\begin{array}{c}
\frac{1}{r_{1}}\left(I_{3}-p_{1} p_{1}^{T}\right) \\
\frac{1}{r_{2}}\left(I_{3}-p_{2} p_{2}^{T}\right) \\
\vdots \\
\frac{1}{r_{n}}\left(I_{3}-p_{n} p_{n}^{T}\right)
\end{array}\right) .
$$

Note that the matrix $\Pi$ depends on the unknown values $r_{i}$. The dynamics of $\delta$ are

$$
\dot{\delta}=\underbrace{\operatorname{diag}(-\operatorname{sk}(\Omega))}_{3 n \times 3 n} \underbrace{\delta}_{3 n \times 1}-\underbrace{\Pi}_{3 n \times 3} \underbrace{V}_{3 \times 1} .
$$

Note that (14) replicates the passivity-like structure seen in (11).

A combination matrix approach [7] is used to combine the full image error $\delta$ into a reduced error

$$
\delta_{1}:=C_{\alpha} \delta, \quad C_{\alpha} \in \mathbb{R}^{3 \times n}
$$

that leads to a nonredundant formulation of the control problem. The combination matrix must be chosen to preserve the passivity-like properties of the image dynamics in the reduced error dynamics. Premultiplying (14) by the combination matrix $C_{\alpha}$ the following two conditions are obtained:

1) $C_{\alpha} \operatorname{diag}(-\operatorname{sk}(\Omega))=-\operatorname{sk}(\Omega) C_{\alpha}, \forall \Omega \in \mathbb{R}^{3}$

2) $C_{\alpha} \Pi=Q_{\alpha}>0$.

Assuming that the matrix $C_{\alpha}$ is full rank, it follows that

$$
C_{\alpha}=\left[\begin{array}{llll}
\alpha_{1} I_{3} & \alpha_{2} I_{3} & \cdots & \alpha_{n} I_{3}
\end{array}\right]
$$

for $\alpha_{i} \geq 0$, for all $i=1, \ldots, n$. Note that $C_{\alpha} \Pi=Q>0$ relies on the fact that $r_{i}>0$. Let $Q_{\alpha}:=C_{\alpha} \Pi$ for a given set of weights $\alpha_{i}$ and note that the exact value of $Q_{\alpha}$ remains unknown! Under and over bounds on $Q_{\alpha}$ are required for the control design in Section IV

$$
\lambda_{\min }^{\alpha} I_{3}<Q_{\alpha}<\lambda_{\max }^{\alpha} I_{3} .
$$

The bounds $\lambda_{\min }^{\alpha}$ and $\lambda_{\max }^{\alpha}$ may be estimated from over and under bounds on the target range $r_{i} i=1, \ldots, n$. These bounds replace the exact estimates of depth information that is necessary to apply existing IBVS algorithms.

Remark III.1: For the class of under-actuated dynamic systems considered, it is physically necessary to use the attitude dynamics to control the orientation of the force input to the linear dynamics in order to stabilize the position of the system. It is impossible to separately stabilize the attitude and position of the camera as is done in classical IBVS. The error criterion proposed regulates only the position of the rigid body. The attitude control is derived from the backstepping errors derived from the proposed error propogated through the system dynamics (cf. Section IV).

It is of interest to consider the reduced error $\delta_{1}$ in closer detail. Recalling (12), it follows that

$$
\delta_{1}=\sum_{i=1}^{n} \alpha_{i} p_{i}-\sum_{i=1}^{n} \alpha_{i} p_{i}^{*} .
$$

Define

$$
q_{\alpha}:=\sum_{i=1}^{n} \alpha_{i} p_{i}
$$

The vector $q_{\alpha} \in \mathbb{R}^{3}$ is the principal visual "feature" that is used in the proposed algorithm. It may be interpreted as a weighted centroid of the observed image. Using centroid information is an old technique in visual servoing [2], [26], [15], [34]. Many of the applications considered that used centroid features involved highly dynamic systems. Historically, this may be due to the simple and fast image processing algorithms needed to extract the centroid of an observed image and consequent improvement in the dynamic response of a closed-loop system based on this information. Although processing limitations are becoming less important in modern applications, centroid image features are still one of the most robust and cheap image features to extract from a scene.

Define

$$
b^{*}:=\sum_{i=1}^{n} \alpha_{i} p_{i}^{*}
$$

Thus, $b^{*} \in \mathcal{A}$ is a known vector with fixed orientation relative to the inertial frame, expressed in the body-fixed-frame. As a 
consequence $b^{*}$ inherits dynamics from the rotational ego-motion of the camera

$$
\dot{b}^{*}=-\Omega \times b^{*} .
$$

Note that the dynamics of $b^{*}$ are independent of the linear velocity of the rigid body since $b^{*}$ is an inertial direction, not a vector position.

The visual error considered may be interpreted as the vectorial distance between a weighted centroid feature and a fixed inertial direction $\delta_{1}=q_{\alpha}-b^{*}$. As a consequence, minimizing $\left|\delta_{1}\right|$ regulates the inertial position of the camera and regulates those degrees of freedom in the attitude dynamics that affect the orientation of the force input $\mathbf{F}$. If there are additional degrees of freedom in the attitude dynamics that are not regulated by the dynamic constraints of the system, then a secondary control error (cf. Sections IV-A and IV-B) may be introduced. If the weighting factors $\alpha$ are chosen to be equal, $\alpha_{i}=\alpha$, $\forall i=1 \ldots n$, then the proposed image feature is co-linear with the accepted image centroid. This particular case is the simplest to implement and has several advantages.

1) It is not necessary to match observed image points directly to desired features. An algorithm based on a construction of this nature will operate without precise target information. As long as the observed target is roughly the same as the expected target then the centroid $q_{\alpha}:=$ $\alpha \sum_{i=1}^{n} p_{i}$ will be a reasonable representation of the true target centroid.

2) The proposed design is not restricted to a finite number of image points $P_{i}$. The proposed error criterion may be extended to solid targets by replacing the summation of separate image points with integration over image surface. Some care must be taken in order that the centroid of the observed target is inversely weighted by the observed area of the image in order that relative depth information is correctly incorporated into the error criterion. The weighted centroid used is

$$
q_{\alpha}:=\frac{\alpha}{\int_{p \in \mathcal{T}}} d p \int_{p \in \mathcal{T}} p d p
$$

using spherical camera geometry and where $\mathcal{T}$ is the area of image as a subset of the camera image surface.

\section{Control Design Methodology}

In this section, an IBVS control derived using robust backstepping techniques [14] for an "eye-in-hand" camera with under-actuated rigid body dynamics.

Deriving $\delta_{1}$ and recalling (16), it follows that

$$
\dot{\delta}_{1}=-C_{\alpha} \operatorname{diag}(\operatorname{sk}(\Omega)) \delta-C_{\alpha} \Pi V .
$$

Recalling (16), Assumption II.2, and the rigid-body dynamics equations (2)-(4), the full dynamics of the error $\delta_{1}$ may be written

$$
\begin{aligned}
\dot{\delta}_{1} & =-\operatorname{sk}(\Omega) \delta_{1}-Q_{\alpha} V \\
m \dot{V} & =-m \Omega \times V+F \\
\dot{R} & =R \operatorname{sk}(\Omega) \\
\mathbf{I} \dot{\Omega} & =-\Omega \times \mathbf{I} \Omega+\Gamma .
\end{aligned}
$$

Initially we consider only the error dynamics equation (20). Define a storage function $S_{1}$ as

$$
S_{1}=\frac{1}{2}\left|\delta_{1}\right|^{2} \text {. }
$$

Taking the time derivative of $S_{1}$ and substituting for (20) yields

$$
\dot{S}_{1}=-\delta_{1}^{T} Q_{\alpha} V .
$$

Note that the passivity-like structure of (20) ensure that (25) is independent of the angular velocity $\Omega$.

As discussed in Section III, the matrix $Q_{\alpha}>0$ is not exactly known, however, the fact that it is positive definite ensures that a choice $V=\delta_{1}$ is sufficient to stabilize $S_{1}$ if the velocity $V$ were available as a control input (kinematic control). The virtual control chosen for (25) is

$$
V^{v}:=\frac{k_{1}}{m} \delta_{1}
$$

where $k_{1}>0$ is a positive constant. If $V \equiv V^{v}$, then $\dot{S}_{1}=$ $-\left(k_{1} / m\right) \delta_{1}^{T} Q_{\alpha} \delta_{1}$ is negative definite in $\delta_{1}$.

With this choice, one has

$$
\dot{\delta}_{1}=-\operatorname{sk}(\Omega) \delta_{1}-\frac{k_{1}}{m} Q_{\alpha} \delta_{1}-\frac{k_{1}}{m} Q_{\alpha} \delta_{2}
$$

where $\delta_{2}$ defines the difference between the desired "virtual control" $V^{v}$ and the true velocity

$$
\delta_{2}:=\frac{m}{k_{1}} V-\delta_{1}
$$

and will form the new error term used in the next step of the backstepping. With the above definitions, one has

$$
\dot{S}_{1}=-\frac{k_{1}}{m} \delta_{1}^{T} Q_{\alpha} \delta_{1}-\frac{k_{1}}{m} \delta_{1}^{T} Q_{\alpha} \delta_{2} .
$$

Deriving $\delta_{2}$ and recalling (21) and (27) yields

$$
\dot{\delta}_{2}=-\operatorname{sk}(\Omega) \delta_{2}+\frac{k_{1}}{m} Q_{\alpha} \delta_{1}+\frac{k_{1}}{m} Q_{\alpha} \delta_{2}+\frac{1}{k_{1}} F .
$$

Let $S_{2}$ be the second storage function using this control algorithm

$$
S_{2}=\frac{1}{2}\left|\delta_{2}\right|^{2} .
$$

Taking the derivative of $S_{2}$, it follows that

$$
\dot{S}_{2}=\frac{k_{1}}{m} \delta_{2}^{T} Q_{\alpha} \delta_{1}+\frac{k_{1}}{m} \delta_{2}^{T} Q_{\alpha} \delta_{2}+\frac{1}{k_{1}} \delta_{2}^{T} F .
$$

The positive semi-definite matrix $Q_{\alpha}$ is not exactly known, however, there are upper and lower bounds on the eigenvalues of $Q_{\alpha}$. Thus, choosing

$$
F:=-\frac{k_{1}^{2} k_{2}}{m} \delta_{2} \quad \text { where } k_{2}>\lambda_{\max }^{\alpha}
$$

is sufficient to stabilize the position of system. Since the rigid-body system considered is under-actuated, the force input $F$ is unable to assign the desired dynamics directly. It is necessary to use the above definition as virtual force inputs in a further stage of the backstepping procedure. Set

$$
F^{v}:=-\frac{k_{1}^{2} k_{2}}{m} \delta_{2} .
$$

A new error term $\delta_{3}$ is defined to measure the scaled difference between the virtual and the true force inputs

$$
\delta_{3}:=\frac{m}{k_{1}^{2} k_{2}}\left(F-F^{v}\right)=\frac{m}{k_{1}^{2} k_{2}} F+\delta_{2} .
$$


The derivative of $\delta_{2}$ of (30) becomes

$$
\dot{\delta}_{2}=-\operatorname{sk}(\Omega) \delta_{2}+\frac{k_{1}}{m} Q_{\alpha} \delta_{1}-\frac{k_{1}}{m}\left(k_{2} I-Q_{\alpha}\right) \delta_{2}+\frac{k_{1}}{m} k_{2} \delta_{3}
$$

and the derivative of the second storage function is now

$$
\dot{S}_{2}=\frac{k_{1}}{m} \delta_{2}^{T} Q_{\alpha} \delta_{1}-\frac{k_{1}}{m} \delta_{2}^{T}\left(k_{2} I-Q_{\alpha}\right) \delta_{2}+\frac{k_{1}}{m} k_{2} \delta_{2}^{T} \delta_{3} .
$$

Deriving $\delta_{3}$ and recalling (30) yields

$$
\begin{aligned}
\dot{\delta}_{3}= & \frac{m}{k_{1}^{2} k_{2}} \dot{F}+\dot{\delta}_{2}-\operatorname{sk}(\Omega) \frac{m}{k_{1}^{2} k_{2}} F+\operatorname{sk}(\Omega) \frac{m}{k_{1}^{2} k_{2}} F \\
= & -\operatorname{sk}(\Omega) \delta_{3}+\frac{k_{1}}{m} Q_{\alpha} \delta_{1}-\frac{k_{1}}{m}\left(k_{2} I-Q_{\alpha}\right) \delta_{2} \\
& +\frac{k_{1}}{m} k_{2} \delta_{3}+\frac{m}{k_{1}^{2} k_{2}}(\dot{F}+\operatorname{sk}(\Omega) F) .
\end{aligned}
$$

Analogously to the previous case and following standard backstepping procedures, let $(\dot{F}+\operatorname{sk}(\Omega) F)^{v}$ denote the virtual control used in the next iteration of the backstepping. The full vectorial term $(\dot{F}+\operatorname{sk}(\Omega) F)^{v}$ is assigned rather than dealing explicitly with the dependence on the actual control inputs. This avoids some complications in the development due to conflicting contributions from both force and torque control inputs. In practice it is a simple matter to compute the actual control inputs from the vectorial control design (cf. Section V). The virtual control assigned is

$$
\frac{m}{k_{1}^{2} k_{2}}(\dot{F}+\operatorname{sk}(\Omega) F)^{v}:=-\frac{\left(k_{1} k_{2}+k_{3}\right)}{m} \delta_{3} .
$$

The expression for the derivative of $\delta_{3}$ may now be written

$$
\begin{aligned}
\dot{\delta}_{3}=-\operatorname{sk}(\Omega) \delta_{3}+\frac{k_{1}}{m} Q_{\alpha} \delta_{1} & -\frac{k_{1}}{m}\left(k_{2} I-Q_{\alpha}\right) \delta_{2} \\
& -\frac{k_{3}}{m} \delta_{3}+\frac{\left(k_{1} k_{2}+k_{3}\right)}{m} \delta_{4} .
\end{aligned}
$$

Here $\delta_{4}$ is the last error term used in the backstepping procedure defined by

$$
\delta_{4}:=\frac{m^{2}}{k_{1}^{2} k_{2}\left(k_{1} k_{2}+k_{3}\right)}\left(\dot{F}+\operatorname{sk}(\Omega) F+\frac{\delta_{3}}{m^{2}}\right) .
$$

Let $S_{3}$ be a third storage function defined by

$$
S_{3}=\frac{1}{2}\left|\delta_{3}\right|^{2} \text {. }
$$

Taking the derivative of $S_{3}$ and recalling (40), one obtains

$$
\begin{array}{r}
S_{3}=\frac{k_{1}}{m} \delta_{3}^{T} Q_{\alpha} \delta_{1}-\frac{k_{1}}{m} \delta_{3}^{T}\left(k_{2} I-Q_{\alpha}\right) \delta_{2}-\frac{k_{3}}{m} \delta_{3}^{T} \delta_{3} \\
+\frac{\left(k_{1} k_{2}+k_{3}\right)}{m} \delta_{3}^{T} \delta_{4} .
\end{array}
$$

The derivative of $\delta_{4}$ is

$$
\begin{aligned}
\dot{\delta}_{4}= & \frac{m^{2}}{k_{1}^{2} k_{2}\left(k_{1} k_{2}+k_{3}\right)}(\ddot{F}+\operatorname{sk}(\dot{\Omega}) F+\operatorname{sk}(\Omega) \dot{F})-\operatorname{sk}(\Omega) \delta_{3} \\
& +\frac{k_{1}}{m} Q_{\alpha} \delta_{1}-\frac{k_{1}}{m}\left(k_{2} I-Q_{\alpha}\right) \delta_{2}-\frac{k_{3}}{m} \delta_{3} \\
& +\frac{\left(k_{1} k_{2}+k_{3}\right)}{m} \delta_{4} .
\end{aligned}
$$

At this stage, the actual control inputs enter into the equations via $\ddot{F}$ and $\dot{\Omega}$. The exact manner in which the control inputs enter depends on each individual application and the arrangements of thrust etc. that generate the force $F$. The application discussed in Section $\mathrm{V}$ indicates the manner in which this calculation is done in practice. It is assumed that the term $m^{2}(\ddot{F}+\operatorname{sk}(\dot{\Omega}) F)$ may be arbitrarily assigned. To achieve the desired control, one chooses

$$
\begin{aligned}
m^{2}(\ddot{F}+\operatorname{sk}(\dot{\Omega}) F) \\
=\frac{k_{1}^{2} k_{2}\left(k_{1} k_{2}+k_{3}\right)}{m} \\
\quad \cdot\left(m \operatorname{sk}(\Omega) \delta_{3}-k_{1} k_{2} \delta_{3}-\left(k_{1} k_{2}+k_{3}+k_{4}\right) \delta_{4}\right) \\
\quad-m^{2} \operatorname{sk}(\Omega) \dot{F} .
\end{aligned}
$$

Substituting the control into the dynamics for $\delta_{4}$ leads to

$\dot{\delta}_{4}=\frac{k_{1}}{m} Q_{\alpha} \delta_{1}-\frac{k_{1}}{m}\left(k_{2} I-Q_{\alpha}\right) \delta_{2}-\frac{\left(k_{1} k_{2}+k_{3}\right)}{m} \delta_{3}-\frac{k_{4}}{m} \delta_{4}$.

Consequently, choosing

$$
S_{4}:=\frac{1}{2}\left|\delta_{4}\right|^{2}
$$

as the final storage function, one obtains

$$
\begin{aligned}
\dot{S}_{4}=\frac{k_{1}}{m} \delta_{4}^{T} Q_{\alpha} \delta_{1}-\frac{k_{1}}{m} \delta_{4}^{T}\left(k_{2} I-Q_{\alpha}\right) \delta_{2} & \\
& -\frac{\left(k_{1} k_{2}+k_{3}\right)}{m} \delta_{4}^{T} \delta_{3}-\frac{k_{4}}{m} \delta_{4}^{T} \delta_{4} .
\end{aligned}
$$

Following the above development, define a candidate Lyapunov function as

$$
\mathcal{L}=S_{1}+S_{2}+S_{3}+S_{4} .
$$

The derivative of $\mathcal{L}$ is

$$
\begin{aligned}
\dot{\mathcal{L}}= & -\frac{k_{1}}{m} \delta_{1}^{T} Q_{\alpha} \delta_{1}+\frac{k_{1}}{m} \delta_{3}^{T} Q_{\alpha} \delta_{1}+\frac{k_{1}}{m} \delta_{4}^{T} Q_{\alpha} \delta_{1} \\
& -\frac{k_{1}}{m} \delta_{2}^{T}\left(k_{2} I-Q_{\alpha}\right) \delta_{2}-\frac{k_{1}}{m} \delta_{3}^{T} Q_{\alpha} \delta_{2} \\
& -\frac{k_{1}}{m} \delta_{4}^{T}\left(k_{2} I-Q_{\alpha}\right) \delta_{2}-\frac{k_{3}}{m} \delta_{3}^{T} \delta_{3}-\frac{k_{4}}{m} \delta_{4}^{T} \delta_{4} .
\end{aligned}
$$

Lemma IV.1: Consider the dynamics defined by (20)-(23). Let $\lambda_{\min }^{\alpha}$ and $\lambda_{\max }^{\alpha}$ be bounds on the maximal and minimal eigenvalues of $Q_{\alpha}$. Let the vectorial controller be given by (45). If the control gains satisfy

$$
\begin{aligned}
& k_{1}>0, \quad k_{2}>\lambda_{\max }^{\alpha}, \\
& k_{3}>k_{1} \lambda_{\max }^{\alpha}\left(\frac{1}{\lambda_{\min }^{\alpha}}+\frac{1}{k_{2}-\lambda_{\min }^{\alpha}}\right)
\end{aligned}
$$

and

$$
k_{4}>k_{1}\left(\frac{\lambda_{\max }^{\alpha}}{\lambda_{\min }^{\alpha}}+\frac{k_{2}-\lambda_{\min }^{\alpha}}{k_{2}-\lambda_{\max }^{\alpha}}\right)
$$

then the error signals

$$
\delta_{1}, \delta_{2}, \delta_{3}, \delta_{4} \rightarrow 0
$$

converge exponentially to zero.

Proof: As the matrix $Q_{\alpha}$ is positive definite, its norm can be lower bounded by $\lambda_{\min }^{\alpha}$. In this case, the Lyapunov function derivative can be bounded by

$$
\begin{aligned}
\dot{\mathcal{L}} \leq & -\frac{k_{1}}{m} \lambda_{\min }^{\alpha} \delta_{1}^{T} \delta_{1}+\frac{k_{1}}{m} \lambda_{\max }^{\alpha} \delta_{3}^{T} \delta_{1}+\frac{k_{1}}{m} \lambda_{\max }^{\alpha} \delta_{4}^{T} \delta_{1} \\
& -\frac{k_{1}}{m}\left(k_{2}-\lambda_{\max }^{\alpha}\right) \delta_{2}^{T} \delta_{2}-\frac{k_{1}}{m} \lambda_{\max }^{\alpha} \delta_{3}^{T} \delta_{2} \\
& -\frac{k_{1}}{m}\left(k_{2}-\lambda_{\min }^{\alpha}\right) \delta_{4}^{T} \delta_{2}-\frac{k_{3}}{m} \delta_{3}^{T} \delta_{3}-\frac{k_{4}}{m} \delta_{4}^{T} \delta_{4} .
\end{aligned}
$$

By completing the square four times to dominate the cross terms, it may be verified that the choice of control gains given 
in the theorem ensures that the right-hand side is negative definite in all the error signals $\delta_{i}, i=1, \ldots, 4$. Classical Lyapunov theory may be applied and the result is proved.

The error coordinates $\delta_{1}$ regulate the position of the camera. The error $\delta_{2}$ regulates the linear velocity of the camera and ensures that it comes to rest. The additional error coordinates $\delta_{3}$ and $\delta_{4}$ incorporate information on the attitude of the camera. This is natural for an under-actuated system since the desired motion can only be obtained by exploiting the attitude dynamics to control the orientation of the force $F$. If the position and linear velocity are regulated then the total external force must be zero, $F=0$. Recalling (5), one has

$$
R \mathbf{F}=e_{3}, \quad u=m g .
$$

It follows that any rotation of the rigid body that would affect the orientation of $\mathbf{F}$ is directly stabilized via the backstepping errors $\delta_{3}$ and $\delta_{4}$.

In certain situations, the errors $\delta_{3}$ and $\delta_{4}$ may provide sufficient information to fully stabilize the attitude of the rigid body. For example, consider the case of an airplane which is tracking a visual target moving with constant velocity (and stationary with respect to an inertial frame chosen to move at the velocity of the target). The proposed control law may be used to design a control that will stabilize the airplane to fly with constant velocity at fixed offset from the moving target. The aerodynamics of the airplane will in turn force the airframe to be aligned in the direction of flight. Thus, in such a case the underlying dynamics of the rigid body motion provide a further stabilizing force that will asymptotically stabilize the attitude of the rigid body. Mathematically, the asymptotic convergence of such a system may be proved by appealing to La Salles principal in the proof of Lemma IV.1.

In other situations, stabilization of the error terms $\delta_{3}$ and $\delta_{4}$ will not determine the full attitude of the device considered. In the case of a helicopter hovering directly over a target, then both pitch and roll components of its attitude are regulated by the errors $\delta_{3}$ and $\delta_{4}$, however, a yaw rotation around the main rotor axis will leave the orientation of $F$ fixed. In the example given in Section V, a simple proportional control is used to stabilize the yaw angular velocity. It may be of interest, however, to stabilize the remaining degree of freedom in the attitude dynamics using an additional error criteria. Two control laws to deal with this situation are presented in Section IV-A and IV-B.

\section{A. Stabilization of Remaining Orientation Dynamics With Reference to Visual Data}

It is often difficult to obtain even a single inertial direction reliably (necessary to define the inertial target vector $b^{*}$ [see (18)]) in order to define the error $\delta_{1}$ [see (15)] and obtaining a second may be impossible. In such a situation, the remaining degree of freedom in the attitude dynamics may be stabilized using only visual information derived from the target.

To control the remaining attitude of a camera via an error in image space, it is necessary to consider error criteria that depend on vector directions fixed in the body fixed frame. A key observation for the proposed control design is that such an error is chosen and minimized after the regulation of the visual error $\delta_{1}$. Thus, regulation of a position is guaranteed independently of the secondary control task involving regulation of the remaining attitude. In this manner, the cascaded control design proposed allows one to overcome some of the pitfalls of visual servoing associated with the coupling of position and attitude errors in the image plane [11].

The desired attitude of the camera may be characterized by specifying a desired inertial orientation for two linearly independent directions in the body fixed frame $\mathcal{A}$. Recalling (5), it is natural to choose $\mathbf{F} \in \mathcal{A}$ as a first unit direction. The following calculations are considerably simpler if the directions chosen are orthogonal. Thus, choose a second vector $\mathbf{G} \in \mathcal{A}$ orthogonal to $\mathbf{F}$ and

$$
\mathbf{H}=\mathbf{G} \times \mathbf{F} .
$$

Two degrees of freedom in the attitude dynamics are fixed by the condition $R \mathbf{F}=e_{3}$ [cf. (51)]. The control objective considered is to align $\mathbf{G}$ as closely as possible with some visual feature extracted from the observed target subject to the constraint $\mathbf{F} \rightarrow e_{3}$. The simplest case is when the weighted centroid feature $q_{\alpha}$ can also be used for stabilization of the attitude. However, if the inertial target direction $b^{*}$ is congruent with the inertial $z$ axis (such as is the case when one wishes to hover directly over a target), then stabilizing the error $\delta_{1}$ leads to the asymptotic relationship

$$
R \mathbf{F}=e_{3}=\frac{R b^{*}}{\left|b^{*}\right|}=\frac{R q_{\alpha}}{\left|q_{\alpha}\right|} .
$$

As a consequence, $q_{\alpha}^{T} \mathbf{G}, q_{\alpha}^{T} \mathbf{H} \longrightarrow 0$ and $q_{\alpha}$ cannot be used as a visual feature to stabilize the remaining degree of freedom in the attitude dynamics. In such a situations it is necessary to define a new "feature" vector computed from image measurements

$$
q_{\beta}=\sum_{i=1}^{i=n} \beta_{i} p_{i}
$$

where $\beta_{i}$ are a set of real constants. It is not required that $\beta_{i}>0$ and differences between observed points may be used to generate $q_{\beta}$ vectors in any direction even if the observed image points are clustered some distance from the camera.

The derivative of $q_{\beta}$ is

$$
\dot{q}_{\beta}=-\operatorname{sk}(\Omega) q_{\beta}-Q_{\beta} V
$$

where

$$
Q_{\beta}:=\sum \frac{\beta_{i}}{r\left(P_{i}\right)} \pi_{p_{i}} .
$$

Define

$$
\sigma_{1}:=\left(\mu-q_{\beta}^{T} \mathbf{G}\right)
$$

where $\mu:=\mu(t)$ is a time-varying scalar evolving according to the O.D.E.

$$
\begin{aligned}
\dot{\mu} & =-a_{1}\left(\mu^{2}-\left|q_{\beta}\right|^{2}+\left\langle q_{\beta}, \mathbf{F}\right\rangle^{2}\right) \\
\mu(0) & :=\left|q_{\beta}(0)\right|^{2}-\left\langle q_{\beta}(0), \mathbf{F}\right\rangle^{2} .
\end{aligned}
$$

The operator $\langle\cdot, \cdot\rangle$ is the scalar product and $a_{1}>0$ is a positive constant. This is a stable first-order nonlinear system driven by a positive autonomous signal $\left|q_{\beta}\right|^{2}-\left\langle q_{\beta}, \mathbf{F}\right\rangle^{2} \geq 0$. Since $\left|q_{\beta}\right|$ is bounded it follows that $\sup \left\{\left|q_{\beta}\right|^{2}\right\} \geq \mu(t)>0$ for all time. It is theoretically possible that $\mu(t) \rightarrow 0$ as $t \rightarrow \infty$ if (and only if) $\operatorname{span}\left\{q_{\beta}\right\} \rightarrow \operatorname{span}\{\mathbf{F}\}$. This possibility is avoided in practice by a suitable choice of $q_{\beta}$. 
Define a cost

$$
W_{1}:=\frac{1}{2} \sigma_{1}^{2}=\frac{1}{2}\left(\mu-q_{\beta}^{T} \mathbf{G}\right)^{2} .
$$

A control is proposed that acts to minimize $W_{1}$. It is shown later that this will achieve the desired control objective. The derivative of $W_{1}$ yields

$$
\dot{W}_{1}=\sigma_{1} q_{\beta}^{T} \mathbf{H F}^{T} \Omega+\sigma_{1}\left(\dot{\mu}+q_{\beta}^{T} \operatorname{sk}(\mathbf{G}) \pi_{\mathbf{F}} \Omega+\mathbf{G}^{T} Q_{\beta} V\right)
$$

where $\pi_{\mathbf{F}}$ is the projection matrix $I_{3}-\mathbf{F F}^{T}$.

Note that assigning $\pi_{\mathbf{F}} \Omega$ corresponds to assigning the two degrees of freedom of $\Omega$ that control the orientation of $\mathbf{F}$. It is exactly these degrees of freedom of $\Omega$ that are fixed by the backstepping error $\delta_{3}$. The component $\mathbf{F}^{T} \Omega$ denotes the remaining degree of freedom in the attitude dynamics. This degree of freedom is decoupled from the rotation $\pi_{\mathbf{F}} \Omega$ assigned by the backstepping procedure and may be used explicitly as a virtual control input for the remaining attitude dynamics.

A complication for the stabilization of the attitude dynamics is that there must always exist a singularity in any stabilizing control defined on a sphere. This singularity usually occurs at the point diametrically opposite to the desired set point. In the proposed design this topological constraint is manifested as a factor $q_{\beta}^{T} \mathbf{H}$ that premultiplies the virtual control $\mathbf{F}^{T} \Omega$ in (54). The term $q_{\beta}^{T} \mathbf{H}$ is zero both at the desired set point ( $q_{\beta}^{T} \mathbf{G}$ maximally positive) and at the antipodal point ( $q_{\beta}^{T} \mathbf{G}$ maximally negative). There are two implications of this property in the control design: Firstly, it is necessary to consider a (globally) discontinuous control in order to drive the system into a neighborhood of the desired set point and avoid unwanted complications due to the antipodal singularity. Secondly, the control design must exploit the relationship between $q_{\beta}^{T} \mathbf{H}$ and $q_{\beta}^{T} \mathbf{G}$ in the limit to obtain exponential convergence. The first difficulty is overcome using a switching control law described below. The second is linked to the role of the time-varying constant $\mu$ in (53).

Let $\epsilon>0$ be a small positive number. Choose a virtual control

$$
\left(\mathbf{F}^{T} \Omega\right)^{v}:= \begin{cases}-a_{1} q_{\beta}^{T} \mathbf{H}, & \text { if }\left\langle q_{\beta}, \mathbf{G}\right\rangle>\epsilon\left|q_{\beta}\right| \\ -\sigma_{1} \frac{\operatorname{sign}\left\langle q_{\beta}, \mathbf{H}\right\rangle}{\left|\left\langle q_{\beta}, \mathbf{H}\right\rangle\right|} \psi, & \text { if }\left\langle q_{\beta}, \mathbf{G}\right\rangle \leq \epsilon\left|q_{\beta}\right|\end{cases}
$$

where the function $\psi=\psi\left(\mu,\left\langle q_{\beta}, \mathbf{G}\right\rangle,\left\langle q_{\beta}, \mathbf{H}\right\rangle\right)>0$ is any real valued function such that for $\left\langle q_{\beta}, \mathbf{G}\right\rangle<-\left|q_{\beta}\right| \epsilon$ (where $\epsilon$ is some small arbitrary number) then $\psi=1$ and such that the switch between the control algorithms is at least once differentiable. The function $\psi$ is a smoothly varying function introduced to allow the backstepping procedure to be undertaken over the entire domain. The details of the convergence on that part of the domain where $\left\langle q_{\beta}, \mathbf{G}\right\rangle<-\left|q_{\beta}\right| \epsilon$ is not of particular interest. It suffices to note that any transient of the system will be forced to converge to the domain $\left\langle q_{\beta}, \mathbf{G}\right\rangle>\left|q_{\beta}\right| \epsilon$ in finite time. Within the domain $\left\langle q_{\beta}, \mathbf{G}\right\rangle>\left|q_{\beta}\right| \epsilon$ it is necessary to analyze the asymptotic convergence properties of the closed-loop system. For $\left\langle q_{\beta}, \mathbf{G}\right\rangle>\left|q_{\beta}\right| \epsilon$, the backstepping error is

$$
\sigma_{2}:=\left(\mathbf{F}^{T} \Omega\right)-\left(\mathbf{F}^{T} \Omega\right)^{v}=\left(\mathbf{F}^{T} \Omega\right)+a_{1} q_{\beta}^{T} \mathbf{H} .
$$

Since the triple $\{\mathbf{F}, \mathbf{G}, \mathbf{H}\}$ of vectors forms a basis for $\mathcal{A}$, then

$$
\left|q_{\beta}\right|^{2}=\left\langle q_{\beta}, \mathbf{F}\right\rangle^{2}+\left\langle q_{\beta}, \mathbf{G}\right\rangle^{2}+\left\langle q_{\beta}, \mathbf{H}\right\rangle^{2}
$$

Using this along with the above derivation, one may write

$$
\begin{aligned}
\dot{W}_{1}=-a_{1} \sigma_{1}^{2}\left(\mu+\left\langle q_{\beta}, \mathbf{G}\right\rangle\right)+\sigma_{1}\left(q_{\beta}^{T} \operatorname{sk}(\mathbf{G}) \pi_{\mathbf{F}} \Omega\right. & \left.+\mathbf{G}^{T} Q_{\beta} V\right) \\
& +a_{1} \sigma_{1} q_{\beta}^{T} \mathbf{H} \sigma_{2} .
\end{aligned}
$$

Here both the translation dynamics and the existing attitude dynamics act as perturbations to the convergence of the storage function $W_{1}$.

A classical backstepping procedure is used to derive the control. Since the switching control used is differentiable, a backstepping procedure generates a valid control over the entire domain (excluding the anti-stable point $q_{\beta}^{T} \mathbf{G}<0$ and $q_{\beta}^{T} \mathbf{H}=0$ ). Only the case where $\left\langle q_{\beta}, \mathbf{G}\right\rangle>\epsilon\left|q_{\beta}\right|$ is studied in detail as this regime will govern the asymptotic behavior of the closed-loop system. On the domain $\left\langle q_{\beta}, \mathbf{G}\right\rangle \leq \epsilon\left|q_{\beta}\right|$ then a suitable control is chosen based on the standard backstepping procedure.

Define

$$
W_{2}:=\frac{1}{2} \sigma_{2}^{2}
$$

Then

$$
\dot{W}_{2}=\sigma_{2}\left(\mathbf{F}^{T} \dot{\Omega}+\mathbf{F}^{T} \operatorname{sk}(\Omega) q_{\beta}-\mathbf{F}^{T} Q_{\beta} V\right)
$$

To simplify the torque control due to the input $\Gamma$, it convenient to linearize the attitude dynamics [see (23)]. Define

$$
w:=-\mathbf{I}^{-1} \Omega \times \mathbf{I} \Omega+\mathbf{I}^{-1} \Gamma .
$$

Since I is full rank, then this is a bijective control input transformation between $\Gamma$ and $w$. With this definition, then $\dot{\Omega}=w$. For $\left\langle q_{\beta}, \mathbf{G}\right\rangle>\epsilon\left|q_{\beta}\right|$, set

$$
\mathbf{F}^{T} w:=-\mathbf{F}^{T} \operatorname{sk}(\Omega) q_{\beta}-\sigma_{1} q_{\beta}^{T} \mathbf{F}-a_{2} \sigma_{2} .
$$

Let

$$
\mathcal{W}=W_{1}+W_{2}
$$

Then, for $\left\langle q_{\beta}, \mathbf{G}\right\rangle>\epsilon\left|q_{\beta}\right|$, one has

$$
\begin{array}{r}
\dot{\mathcal{W}}=-a_{1} \sigma_{1}^{2}\left(\mu+\left\langle q_{\beta}, \mathbf{G}\right\rangle\right)+\sigma_{1}\left(q_{\beta}^{T} \operatorname{sk}(\mathbf{G}) \pi_{\mathbf{F}} \Omega-\mathbf{G}^{T} Q_{\beta} V\right) \\
-a_{2} \sigma_{2}^{2}-\sigma_{2} \mathbf{F}^{T} Q_{\beta} V .
\end{array}
$$

Theorem IV.2: Consider the system dynamics equations (20)-(23) and let the force $F$ be given by (5). Let $\lambda_{\min }^{\alpha}$ and $\lambda_{\max }^{\alpha}$ be bounds on the maximal and minimal eigenvalues of $Q_{\alpha}$ and $\lambda_{\max }^{\beta}$ be a bound on the maximal eigenvalue of $Q_{\beta}$. Let $1>\epsilon>0$ be a suitably small positive constant. Let the vectorial controller be given by (45). Set the remaining degree of freedom in the torque control to be given by (58) along with the control transformation (57) for $\mathbf{G}^{T} q_{\beta}>\epsilon\left|q_{\beta}\right|$ and the standard backstepping control law for $\mathbf{G}^{T} q_{\beta} \leq \epsilon\left|q_{\beta}\right|$. Choose gains $k_{1}>\epsilon$, then

$$
k_{2}>\lambda_{\max }^{\alpha}+1, \quad k_{3}>\frac{9 k_{1}\left(\lambda_{\max }^{\alpha}\right)^{2}}{\left(k_{2}-\lambda_{\max }^{\alpha}\right)}
$$

and

$$
k_{4}>\frac{9 k_{1}\left(k_{2}-\lambda_{\min }^{\alpha}\right)^{2}}{\left(k_{2}-\lambda_{\max }^{\alpha}\right)} .
$$


Set

$$
f_{1}:=\frac{k_{1}\left(\lambda_{\max }^{\beta}\right)^{2}}{m}, \quad f_{2}:=\frac{2 k_{1}^{2} k_{2}\left(k_{1} k_{2}+k_{3}\right)}{m^{3} g}
$$

and choose

$$
\begin{aligned}
& a_{1}>\max \left\{\frac{15 f_{1}}{2 \epsilon \lambda_{\min }^{\alpha}}, \frac{15 f_{1}}{2 \epsilon\left(k_{2}-\lambda_{\max }^{\alpha}\right)}, \frac{15 f_{2}^{2}}{4 \epsilon m^{3} k_{3}}, \frac{15 f_{2}^{2}}{4 \epsilon m k_{4}}\right\} \\
& a_{2}>\max \left\{\frac{9 f_{1}}{2 \lambda_{\min }^{\alpha}}, \frac{9 f_{1}}{2\left(k_{2}-\lambda_{\max }^{\alpha}\right)}\right\} .
\end{aligned}
$$

Assume that the thrust magnitude

$$
u>\frac{1}{2} m g
$$

is always at least half the gravitational force and that the visual feature $q_{\beta}$ satisfies

$$
\left|q_{\beta}\right| \geq \epsilon \text {. }
$$

Then the error coordinates $\delta_{1}$ [see (15)], $\delta_{2}$ [see (28)], $\delta_{3}$ [ see (15)], $\delta_{4}$ [see (28)], and $\sigma_{1}$ and $\sigma_{2}$ converge exponentially to zero. Furthermore,

$$
\left\langle q_{\beta}, \mathbf{G}\right\rangle \rightarrow+\sqrt{\left|q_{\beta}\right|^{2}-\left\langle q_{\beta}, \mathbf{F}\right\rangle} .
$$

Sketch of Proof: It may be directly verified that the switching control chosen will drive the system to satisfy the condition $\left\langle q_{\beta}, \mathbf{G}\right\rangle>\epsilon\left|q_{\beta}\right|$ in finite time. Since the possible perturbations due to the linear dynamics are exponentially decaying, then there exists a finite time $T_{1}$ such that for all $t>T_{1}$ one may assume $\left\langle q_{\beta}, \mathbf{G}\right\rangle>\epsilon\left|q_{\beta}\right|$. Define

$$
\mathcal{U}:=\mathcal{L}+\mathcal{W}
$$

Premultiplying (41) by $\pi_{\mathbf{F}}$, using (5), and bounding the thrust $u \geq 1 / 2 m g$ yields

$$
\begin{aligned}
\left|\pi_{\mathbf{F}} \Omega\right| & \leq \frac{\left(k_{1}^{2} k_{2}\left(k_{1} k_{2}+k_{3}\right)\right)}{u m^{2}}\left|\frac{\pi_{\mathbf{F}} \delta_{3}}{m^{2}}-\pi_{\mathbf{F}} \delta_{4}\right| \\
& \leq \frac{2\left(k_{1}^{2} k_{2}\left(k_{1} k_{2}+k_{3}\right)\right)}{m^{3} g}\left(\frac{1}{m^{2}}\left|\delta_{3}\right|+\left|\delta_{4}\right|\right) .
\end{aligned}
$$

From (28), one has

$$
|V| \leq \frac{k_{1}}{m}\left(\left|\delta_{2}\right|+\left|\delta_{1}\right|\right)
$$

Substituting these expressions into (59), one obtains bilinear cross terms between the errors $\sigma_{1}$ and $\sigma_{2}$ and the errors $\delta_{1}, \ldots, \delta_{4}$. The value of $\dot{\mathcal{U}}$ may be calculated from (50) and (59). To ensure that all the cross terms are dominated, it is simply a case of completing the square ten times. The approach taken was to divide the negative definite terms in $\left|\delta_{i}\right|^{2}$ into three parts, the first to provide a negative definite term for the final stability argument, the second to dominate the cross terms $\delta_{i}^{T} \delta_{j}$ for $i \neq j$, and the third part divided equally to help dominate the cross terms $\sigma_{i}\left|\delta_{j}\right|$. The term $-a_{1} \epsilon \sigma_{1}^{2}$ was divided into five parts to cover cross terms in the errors $\left\{\left|\delta_{1}\right|, \ldots,\left|\delta_{4}\right|\right\}$ and leave a part negative definite while the term $-a_{2} \sigma_{2}^{2}$ need only be divided in three. It is from this procedure that the bounds stated in the Theorem are obtained (note the difference from Lemma IV.1 due to the need to use some control action in the linear dynamics to bound perturbations in the attitude dynamics). The full procedure leads to

$$
\dot{\mathcal{U}} \leq-a \mathcal{U}
$$

where

$$
c=\min \left\{\frac{\epsilon \lambda_{\min }^{\alpha}}{3 m}, \frac{\epsilon}{3 m}, \frac{9 k_{1}\left(\lambda_{\max }^{\alpha}\right)^{2}}{3 m}, \frac{9 k_{1} k_{2}^{2}}{3 m}, \frac{a_{1} \epsilon}{5}, \frac{a_{2}}{5}\right\} .
$$

It follows from classical Lyapunov theory that the errors $\delta_{1}, \ldots, \delta_{4}, \sigma_{1}, \sigma_{2}$ converge to zero exponentially.

Furthermore, from La Salles principal $\mu$ converges to an invariant set. Firstly, $\sigma_{1}=0$ implies $\mu=\left\langle q_{\beta}, \mathbf{G}\right\rangle \geq 0$. Secondly, it is easily verified that $\dot{\mu}$ converges to zero and hence

$$
\mu^{2}=\left|q_{\beta}\right|^{2}-\left\langle q_{\beta}, \mathbf{F}\right\rangle^{2}
$$

asymptotically. Combining these two points it follows that $\left\langle q_{\beta}, \mathbf{H}\right\rangle=0$ in the limit. The result follows.

Theorem IV.2 provides a complete characterization of the stabilization of the body fixed frame. The final orientation in alignment is achieved by noting that the conditions of Theorem IV. 2 result in

$$
q_{\beta} \in \operatorname{span}\{\mathbf{G}, \mathbf{F}\}
$$

and $\left\langle q_{\beta}, \mathbf{G}\right\rangle \geq 0$. Thus, specifying a suitable attitude is simply a case of choosing a suitable vector $q_{\beta}$ based on a priori knowledge of the target and desired set point pose. An important observation is that the constant $\epsilon$ used in the proof of Theorem IV.2 is the worst case lower bound on the control margin available to stabilize the remaining attitude dynamics. In practice, there will be considerably more control authority than this available for the asymptotic convergence of the system. Thus, the estimate of the exponential stability constant $c>0$ derived in the theorem is likely to be considerably smaller than the true stability margin for the system. Finally, it should be mentioned that Theorem IV.2 is intended as a proof of concept and the that the gains used are not optimized. In practice, the gains would be optimized based on the particular configuration considered. A gain schedule based on estimated values of $\left\{\lambda_{\max }\left(Q_{\alpha}\right), \lambda_{\min }\left(Q_{\alpha}\right)\right.$, $\left.\lambda_{\max }\left(Q_{\beta}\right)\right\}$ may be used to improve the overall performance of the closed-loop system.

\section{B. Stabilization of Remaining Orientation Dynamics with Reference to an Inertial Measurement}

If a second inertial direction is available (similar in nature to $\left.b^{*}\right)$, then there is no need to resort to visual data to regulate the attitude and a simple error based on the state rotation matrix may be used.

Denote the new inertial direction by $c_{0}^{*} \in \mathcal{I}$ and assume without loss of generality that $\left|c_{0}^{*}\right|=1$ and that $\left\langle c_{0}^{*}, e_{3}\right\rangle=0$. This guarantees that, in the limit $\left\langle\mathbf{F}, c_{0}^{*}\right\rangle=0$. As a consequence, it is unnecessary to introduce a scaling factor such as $\mu$ [cf. (53)] and the error considered is simply

$$
\gamma:=1-\left\langle R^{T} c_{0}^{*}, \mathbf{G}\right\rangle
$$

where $\mathrm{G}$ is a fixed direction in $\mathcal{A}$, the body fixed frame orthogonal to the direction $\mathbf{F}$. 
Apart from a slightly simpler form due to the fact that $\gamma$ does not depend on visual data, the derivation of the attitude control law is analogous to that presented in Section IV-A. Note that the attitude control in this case will only depend on the signals $\delta_{3}, \delta_{4}$ since the $\delta_{1}, \delta_{2}$ errors only entered into Theorem IV.2 due to the derivative of $q_{\beta}$. It is still necessary to use the stability of the position dynamics to prove stability of the final attitude dynamics. The details are somewhat tedious and are omitted due to space constraints.

\section{Application to a Scale Model AUTONOMOUS HELICOPTER}

In this section, the procedure presented in Section IV is applied to an idealized model of the dynamics of a scale model autonomous helicopter.

An important control task for an autonomous helicopter is to achieve stable hover over a target. This task forms the first stage of any landing maneuver. Rough position may be obtained from a GPS unit but accurate positioning must be done using local measurement systems such as a vision system. The remaining system variables required for the proposed control design may be derived from external systems. For example, the velocity $V$ may be derived from an extended Kalman filter based on differential GPS data and accelerometer data. The angular velocity may be obtained by a "three axis rate gyro" assembly while a three axis linear accelerometer, along with some basic filtering, will give a good approximation of the gravitational direction. The gravitational direction is the only inertial direction that is necessary for the proposed control.

A helicopter has four control inputs available.

- $u e_{3}$ : The principal rotor provides a strong lift force termed "heave," oriented along the vertical axis of the helicopter body frame. It is the main force responsible for sustaining the helicopter in flight and used for forward propulsion.

- $\Gamma$ : Torque control for the attitude dynamics including the aerodynamic effects is obtained via the tail rotor collective pitch and cyclic pitch to the main rotor.

A helicopter has six degrees of freedom, the dynamic model has twelve states, and there are only four control inputs. The idealized dynamics of a model helicopter expressed in terms of the motion of a target in the visual plane are given by (20)-(23) with the force defined by

$$
F=-u e_{3}+m g R^{T} e_{3}
$$

where $m$ is the total mass of the helicopter and $g$ is the gravitational constant. For more details on the modeling procedure, the interested reader is directed to any number of texts on helicopter modeling and control (cf., for example, [23]).

For the case of an idealized helicopter [see (60) and full torque control] then the control law defined in (45) becomes

$$
\begin{array}{r}
\left(-\ddot{u} e_{3}+\operatorname{sk}(\dot{\Omega}) e_{3} u\right)=\operatorname{sk}(\Omega) \dot{u} e_{3}+\frac{k_{1}^{2} k_{2}\left(k_{1} k_{2}+k_{3}\right)}{m^{2}} \\
\cdot\left(\operatorname{sk}(\Omega) \delta_{3}-\frac{k_{1} k_{2}}{m} \delta_{3}-\frac{\left(k_{1} k_{2}+k_{3}+k_{4}\right)}{m} \delta_{4}\right)
\end{array}
$$

Note that the second derivative of the heave control $\ddot{u}$ enters into this expression. To implement this design procedure, it is necessary to dynamically extend the heave control

$$
\ddot{u}:=v
$$

where $v$ is a new control input and the dynamics $\ddot{u}=v$ are computed within the control structure. Using the control input transformation (57), the dynamics equation (62), and knowing that $\operatorname{sk}\left(e_{3}\right)$ is of rank two with entries only in the first and second columns, one obtains

$$
v e_{3}+u \operatorname{sk}(\dot{\Omega}) e_{3}=\left(\begin{array}{c}
u w_{2} \\
-u w_{1} \\
v
\end{array}\right) .
$$

It remains only to observe that, as long as $u \neq 0$, the control signals $w_{1}, w_{2}$, and $v$ are uniquely determined by the visual servo control equation (45). This is certainly the case in hover conditions since $u \approx m g$ must counteract the gravitational force.

The above control design leaves $w_{3}=e_{3}^{T} w$ free to stabilize the yaw angle to a desired value. In this example, a simple proportional stabilizing feedback

$$
w_{3}:=-K \Omega_{3}
$$

for $K>0$, a suitable constant, is applied. Due to the decoupled nature of the attitude dynamics (using the transformed control $w)$, one has

$$
\frac{d}{d t}\left|\Omega_{3}\right|^{2}=-K\left|\Omega_{3}\right|^{2}
$$

and Lyapunov theory ensures that the yaw velocity converges to zero and that the helicopter (at least the simulated helicopter) will stabilize in hover using visual data.

\section{A. Simulation}

In this subsection, a simulation is presented to illustrate the performance of the proposed control law. The simulation considers the case of stabilization of the helicopter already in hover flight to a new set point several meters distance from the initial condition. Consider the case where one wishes to position the camera parallel to a plan target characterized by a square. In the case of a pin-hole camera, the visual measurements available are the projective coordinates of the four points defining the square $\left\{\left(u_{1}, v_{1}\right),\left(u_{2}, v_{2}\right),\left(u_{3}, v_{3}\right),\left(u_{4}, v_{4}\right)\right\}$. These coordinates are transformed into spherical coordinates using the transformation equation (10). The desired target vector $b^{*}$ is chosen such that the camera set point is located $6 \mathrm{~m}$ above the square. It is defined by $\{(-a, a),(a, a),(a,-a),(-a,-a)\}$ where $a$ represents the ratio between the vertex length and the final desired range. In this experiment the parameter $a$ has been chosen to be equal to 0.4851 . The weights $\alpha_{i}$ are all taken to be equal to unity. Using the above specification, the desired feature $b^{*}$ will be defined in the body fixed frame as follows:

$$
b^{*}=R^{T}\left(0,0, \frac{4}{\sqrt{1+2 a^{2}}}\right) .
$$

The parameters used for the dynamic model are based on preliminary measurements for a VARIO $23 \mathrm{cc}$ scale model helicopter owned by HeuDiaSyC (CNRS Laboratory, Université de Technologie de Compiègne). The values used are $m=9.6$, $I=\operatorname{diag}(0.4,0.56,0.22)$ and $g=9.8$. The magnitude of the 


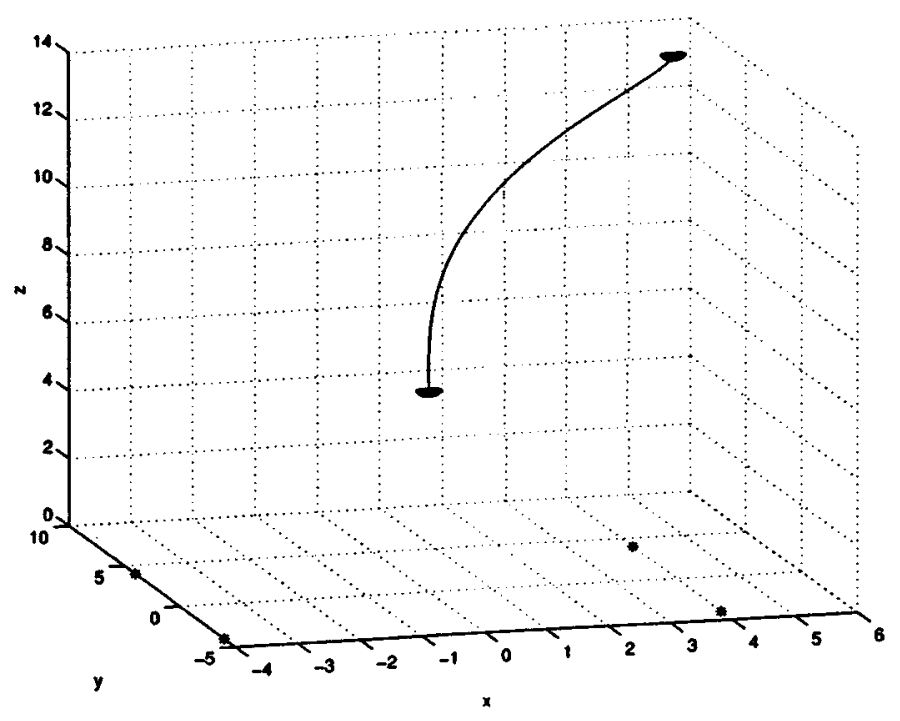

Fig. 3. Positioning of the helicopter with respect to the target.
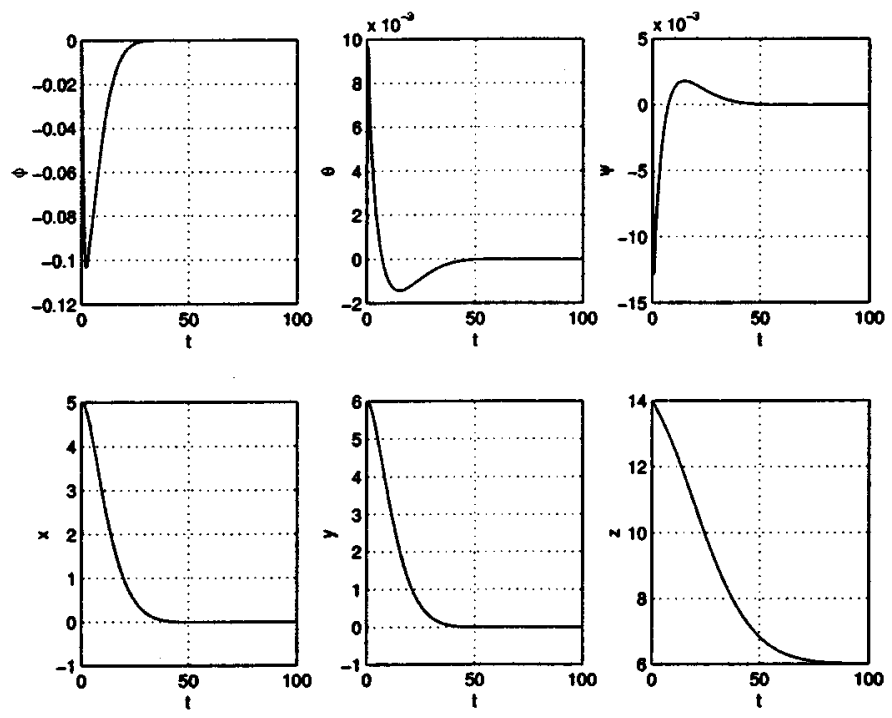

Fig. 4. Evolution of the attitude and position of the helicopter in "roll, pitch, and yaw" Euler angles and Cartesian space coordinates.

initial force input is chosen to be $u_{0}=g m \approx 94$, corresponding to the fact that the helicopter is initially in hover flight. The initial position is

$$
\xi_{0}=\left(\begin{array}{c}
5 \\
6 \\
-14
\end{array}\right), \quad V_{0}=\Omega_{0}=0, \quad \text { and } \quad \phi_{0}=0 .
$$

The center of the target $\hat{\xi}$ is chosen to be the origin of the inertial frame.

Assuming that $\lambda_{\min }\left(Q_{\alpha}\right)$ is a function of initial conditions and that $\lambda_{\max }\left(Q_{\alpha}\right)$ is a function of the desired location, i.e., $\left|\delta_{1}\right|$ is always decreasing, we choose

$$
\lambda_{\min }^{\alpha}=0.02 \text { and } \lambda_{\max }^{\alpha}=0.37 \text {. }
$$

From the assumed values of $\lambda_{\min }^{\alpha}$ and $\lambda_{\max }^{\alpha}$, we have used the following control gains: $k_{1}=0.2, k_{2}=1, k_{3}=3$, and $k_{4}=3$ that satisfy the conditions of Lemma IV.1.

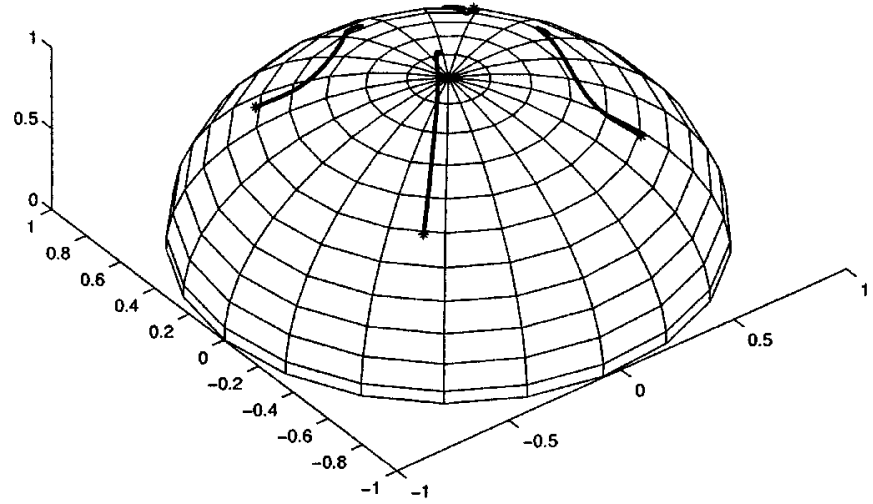

Fig. 5. Evolution of the image features in the spherical image space.

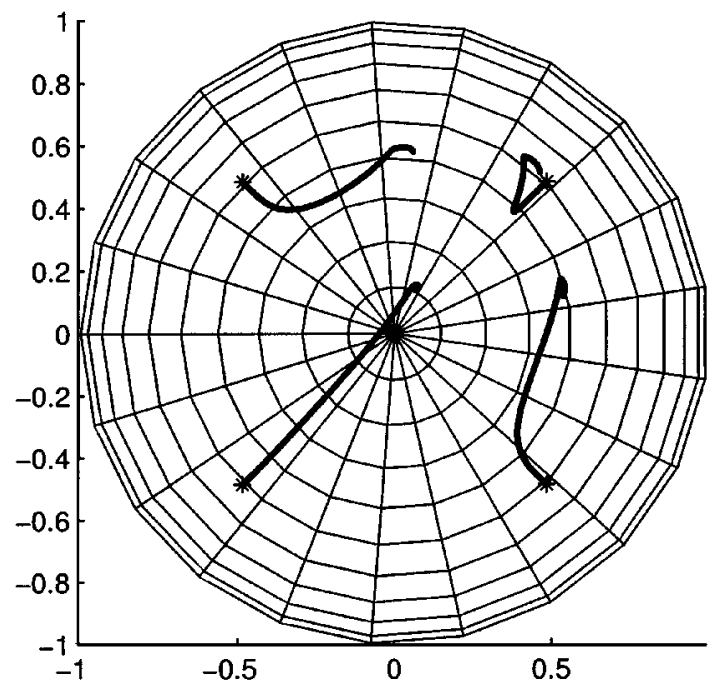

Fig. 6. Evolution of the image features in the image plan.

Simulation results of the closed-loop behavior of the idealized helicopter model considered equipped with the proposed control are shown in Figs. 3-6.

\section{CONCLUSION}

This paper has provided a rigorous derivation of an IBVS control design for a class of under-actuated dynamic systems. The algorithm is novel in considering the full dynamics of the rigid-body motion of the camera fixed frame and leading to a control design in which only bounds on the relative depths of the image points are required. An interesting aspect of the design is the use of the spherical projection for the camera geometry. The simulation of the control of a scale-model autonomous helicopter shows the nature of potential applications of this work.

The authors would like to acknowledge the important issue associated with the discrete sampling involved in using any camera system. Corke et al. [4] showed that the dynamics associated with the camera sampling rate in a sampled-data visual servo system may lead to significant performance degradation and even instability of an IBVS closed-loop system. This issue is beyond the scope of the present paper or indeed most of the recent work in visual servoing. This issue must not be ignored in any real-world application of visual servo systems. 


\section{ACKNOWLEDGMENT}

The authors would like to thank P. Corke and F. Chaumette for many interesting and informative discussions. The authors would also like to thank the anonymous reviewers of the original version of this paper who provided comments and remarks that have been invaluable in helping us understand what we are trying to do as well as significantly improving the paper.

\section{REFERENCES}

[1] O. Amidi, T. Kanade, and R. Miller, "Robust vision for vision-based control of motion," in Vision-Based Autonomous Helicopter Research at Carnegie Mellon Robotics Institute (1991-1998), M. Vincze and G. D. Hager, Eds. New York: IEEE Press and SPIE Optical Engineering Press, 1999, ch. 15, pp. 221-232.

[2] R. L. Andersson, A Robot Ping-Pong Player: Experiment in Real-Time Intelligent Control. Cambridge, MA: MIT Press, 1988.

[3] J. Chahl and M. Srinivasan, "Panoramic vision system for imaging, ranging and navigation in three dimensions," in Proc. Int. Conf. Field and Service Robotics, FSA'99, Pittsburgh, PA, 1999.

[4] P. Corke and M. Good, "Dynamic effects in visual closed-loop systems," IEEE Trans. Robot. Automat., vol. 12, pp. 671-684, Oct. 1996.

[5] P. Corke and S. A. Hutchinson, "A new partitioned approach to imagebased visual servo control," in Proc. Int. Symp. Robotics, Montreal, ON, Canada, May 2000.

[6] K. Deguchi, "Optimal motion control for image-based visual servoing by decoupling translation and rotation," in Proc. Int. Conf. Intelligent Robots and Systems, 1998, pp. 705-711.

[7] B. Espiau, F. Chaumette, and P. Rives, "A new approach to visual servoing in robotics," IEEE Trans. Robot. Automat., vol. 8, pp. 313-326, June 1992.

[8] H. Goldstein, Classical Mechanics, 2nd ed. Reading, MA: AddisonWesley, 1980, Addison-Wesley Series in Physics.

[9] R. Horaud, B. Conio, O. Leboulleux, and B. Lacolle, "An analytic solution for the perspective 4-point problem," in Computer Vision, Graphics and Image Processing. New York: Academic , 1989.

[10] K. Hosada and M. Asada, "Versatile visual servoing without knowledge of true Jacobian," in Proc. IEEE/RSJ Int. Conf. Intelligent Robots and Systems, Munich, Germany, 1994, pp. 186-193.

[11] S. Hutchinson, G. Hager, and P. Corke, "A tutorial on visual servo control," IEEE Trans. Robot. Automat., vol. 12, pp. 651-670, Oct. 1996.

[12] R. Kelly, "Robust asymptotically stable visual servoing of planar robots," IEEE Trans. Robot. Automat., vol. 12, pp. 759-766, Oct. 1996.

[13] T. J. Koo and S. Sastry, "Output tracking control design of a helicopter model based on approximate linearization," in Proc. IEEE Conf. Decision and Control CDC'98, 1998.

[14] M. Krstic, I. Kanellakopoulos, and P. V. Kokotovic, Nonlinear and Adaptive Control Design. Providence, RI: Amer. Math. Soc., 1995.

[15] M. Lei and B. K. Ghosh, "Visually guided robotic motion tracking," in Proc. Thirteenth Annu. Conf. Communication, Control and Computing, 1992, pp. 712-721.

[16] R. Mahony, T. Hamel, and A. Dzul, "Hover control via approximate lyapunov control for a model helicopter," in Proc. 1999 Conf. Decision and Control, Phoenix, AZ, 1999.

[17] E. Malis, F. Chaumette, and S. Boudet, "2-1/2-d visual servoing," IEEE Trans. Robot. Automat., vol. 15, pp. 238-250, Apr. 1999.

[18] A. Maruyama and M. Fujita, "Visual feedback control of rigid body motion based on dissipation theoretical approach," in Proc. 38th Conf. Decision and Control, Phoenix, AZ, 1999, pp. 4161-4166.

[19] G. Morel, T. Liebezeit, J. Szewczyk, S. Boudet, and J. Pot, "Explicit incoporation of 2D constraints in vision based control of robot manipulators," in Robust Vision for Vision-Based Control of Motion, P. Corke and J. Trevelyan, Eds. New York: Springer-Verlag, 1999, vol. 250, Lecture Notes in Control and Information Sciences, pp. 99-108.

[20] N. Papanikolopoulos, P. Khosla, and T. Kanade, "Adaptive robot visual tracking," in Proc. Amer. Control Conf., 1991, pp. 962-967.

[21] J. A. Piepmeier, "A dynamic quasi-Newton method for model independent visual servoing," Ph.D. dissertation, Georgia Inst. Technology, Atlanta, July 1999.

[22] R. Pissard-Gibollet and P. Rives, "Applying visual servoing techniques to control of a mobile hand-eye system," in Proc. IEEE Int. Conf. Robotics and Automation, ICRA'95, Nagasaki, Japan, 1995, pp. 166-171.

[23] R. W. Prouty, Helicopter Performance, Stability and Control. Melbourne, FL: Krieger, 1986 (reprinted with additions 1995).

[24] P. Rives, P. Bouthémy, B. Prasada, and E. Dubois, "Recovering the orientation and the position of a rigid body in space from a single view," INRS-Telecommunications, Verdun, QC, Canada, Tech. Rep., 1981.
[25] C. Samson, M. Le Borgne, and B. Espiau, Robot Control: The Task Function Approach. Oxford, U.K.: Oxford Univ. Press, 1991.

[26] A. Sanderson, L. Weiss, and C. Neuman, "Dynamic sensor based control of robots with visual feedback," IEEE Trans. Robot. Automat., vol. RA-3, pp. 404-417, 1987.

[27] O. Shakernia, Y. Ma, T. J. Koo, and S. Sastry, "Landing an unmanned air vehicle: Vision based motion estimation and nonlinear control," Asian J. Contr., to be published.

[28] H. Shim, T. J. Koo, F. Hoffmann, and S. Sastry, "A comprehensive study of control design for an autonomous helicopter," in Proc. 37th Conf. Decision and Control CDC'98, 1998.

[29] A. Sira-Ramirez and R. Castro-Linares, "On the regulation of a helicopter system: A trajectory planning approach for the Liovillian model," in Proc. Eur. Control Conf. ECC'99, Karlsruhe, Germany, 1999, Session DM-14.

[30] M. Srinivasan, J. Chahl, K. Weber, S. Venkatesh, M. Nagle, and S. Zhang, "Robot navigation inspired by principles of insect vision," Robot Autonomous Syst., vol. 26, pp. 203-216, 1999.

[31] L. E. Weiss, A. C. Sanderson, and C. P. Neuman, "Dynamic sensor-based control of robots with visual feedback," IEEE Trans. Robot. Automat., vol. RA-3, pp. 404-417, Oct. 1987.

[32] D. Wettergreen, C. Gasket, and A. Zelinsky, "Autonomous guidance and control for an underwater robotic vehicle," in Proc. Int. Conf. Field and Service Robotics FSR'99, Piitsburgh, PA, 1999.

[33] W. J. Wilson, C. C. W. Hulls, and G. S. Bell, "Relative end-effector conrol using Cartesian position-based visual servoing," IEEE Trans. Robot. Automat., vol. 12, pp. 684-696, Oct. 1996.

[34] B. Yoshimi and P. K. Allen, "Active, uncalibrated visual servoing," in Proc. IEEE Int. Conf. Robotics and Automation, ICRA'94, San Diego, CA, 1994, pp. 156-161.

[35] E. Zergeroglu, D. Dawson, M. de Queiroz, and S. Nagarkatti, "Robust visual-servo control of robot manipulators in the presence of uncertainty," in Proc. 38th Conf. Decision and Control, Phoenix, AZ, 1999.

[36] H. Zhang and J. P. Ostrowski, "Visual servoing with dynamics: Control of an unmanned blimp," in Proc. IEEE Int. Conf. Robotics and Automation, Detroit, MI, 1999, pp. 618-623.

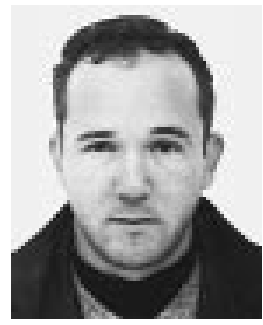

Tarek Hamel received the B.Eng. degree from the Institut d'Electronique et d'Automatique d'Annaba, Algeria, in 1991 and the Ph.D. degree in robotics from the University of Technologie of Compiègne in 1995.

He conducted his Ph.D. research at the Laboratoire Heuristique et Diagnostic des systemes complexes de Compiegne, France. After two years as a Research Assistant at the Compiegne University of Technology, France, he joined the Centre d'Etudes de Mecanique d'Iles de France in 1997 as an Assistant Professor. His research interests include control theory and robotics with particular focus on nonlinear control and vision-based control. He is involved in applications of these techniques to the control of unmanned aerial vehicles and mobile robots.

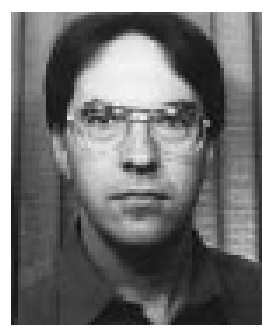

Robert Mahony received the science degree in applied mathematics and geology and the Ph.D. degree in systems engineering from the Australian National University, Canberra, in 1989 and 1994, respectively.

After working for a year as a geophysicist processing marine seismic data he returned to pursue his doctoral degree at ANU. Between 1994 and 1997, he worked as a Research Fellow in the Cooperative Research Centre for Robust and Adaptive Systems based in the Research School of Information Sciences and Engineering, Australian National University, Australia. From 1997 to 1999, he held a post as a Post-Doctoral Fellow in the CNRS Laboratory for Heuristics Diagnostics and complex systems (Heudiasyc), Compiegne University of Technology, France. Between 1999 and 2001 he held a Logan Fellowship in the Department of Engineering and Computer Science at Monash University, Melbourne, Australia. Since July 2001, he has held the post of Senior Lecturer in mechatronics at the Department of Engineering, ANU. His research interests are in nonlinear control theory with applications in mechanical systems and motion systems, and mathematical systems theory and geometric optimization techniques with applications in linear algebra and digital signal processing. 